\title{
Characterization and identification of surface crystals on smear-ripened cheese by polarized light microscopy
}

\author{
P. J. Polowsky, ${ }^{*}$ G. F. Tansman, ${ }^{*}$ P. S. Kindstedt, ${ }^{* 1}$ and J. M. Hughes $†$ \\ *Department of Nutrition and Food Sciences and \\ †Department of Geology, University of Vermont, Burlington 05405
}

\section{ABSTRACT}

Surface crystallization and radial demineralization of $\mathrm{Ca}, \mathrm{P}$, and $\mathrm{Mg}$ occur in smear-ripened cheese. Furthermore, crystals of ikaite, struvite, calcite, and brushite have been identified in cheese smears by powder X-ray diffractometry (PXRD), and ikaite and struvite exist in smears as single crystals. Polarized light microscopy (PLM) is a simple, inexpensive, and well-established method in geology to detect and identify single crystals. However, use of PLM to identify cheese crystals has not been reported previously. The specific objectives of this research were (1) to identify crystals in cheese smears using selected PLM criteria; (2) to compare identification by PLM against PXRD; and (3) to develop and evaluate a novel treatment for smear material to improve crystal analyses by both PLM and PXRD. Duplicate wheels of 4 cheeses produced by different manufacturers were obtained from retail sources. Scrapings of surface smears were prepared and analyzed by PLM and PXRD by previously described methods. Crystals were categorized by PLM based on angle of extinction (AE), birefringence behavior under crossed polarizers and quartz filters, and size and shape (circularity) by image analysis. Crystals observed by PLM fell almost exclusively into 2 readily differentiated groups based on birefringence behavior and estimated angle of extinction. Group $1(\mathrm{n}=18)$ were highly birefringent with $\mathrm{AE}=88^{-92^{\circ}}$, whereas group $2(\mathrm{n}=28)$ had no birefringence with $\mathrm{AE}=13-26^{\circ}$. Group 2 crystals were significantly larger and more circular than group 1 crystals. Group 1 and 2 were identified as struvite and ikaite, respectively, based on known birefringence and AE characteristics. Struvite was identified in all 4 cheeses by PLM but in only 3 cheeses by PXRD. Ikaite was identified in 3 cheeses by

Received March 7, 2018.

Accepted May 2, 2018.

${ }^{1}$ Corresponding author: Paul.Kindstedt@uvm.edu
PLM but in only 2 cheeses by PXRD. These discrepancies occurred because the smear scrapings from 1 cheese contained excessive amorphous matter that caused extreme background noise, potentially obscuring diffractogram peaks that may have been present. To minimize noise, smear scrapings were dispersed in aqueous $\mathrm{NaOH}$ ( $\mathrm{pH}$ 10) before analyses, which resulted in consistent results by PXRD and PLM. The method also rendered high-quality images by PLM. Data suggest that PLM may offer a simple and inexpensive means to identify struvite, ikaite, and possibly other single crystals in cheese smears.

Key words: cheese, crystal, polarized light microscopy

\section{INTRODUCTION}

Crystallization of calcium phosphate at the surface of soft white mold surface ripened cheeses, such as Brie and Camembert, sets up pronounced outward migration of endogenous cheese minerals, such as calcium, magnesium, and phosphorus, during ripening (Le Graet et al., 1983; Karahadian and Lindsay, 1987; Le Graet and Brule, 1988; Tansman et al., 2017a). The surface crystals chelate minerals and deplete the water phase of mineral components, which in turn establishes a concentration gradient with net mineral migration outward that fuels further crystallization. This process leads to demineralization of the curd body and results in $\mathrm{pH}$-dependent radial softening, significantly affecting the overall texture and body of the resulting cheese (Metche and Fanni, 1978). A similar process of radial softening occurs in soft, surface-ripened, washed-rind (smear-ripened) cheeses. However, the surface crystals that form on soft, washed-rind cheese consist mostly of ikaite (calcium carbonate hexahydrate), struvite (magnesium ammonium phosphate hexahydrate), and small amounts of calcite (calcium carbonate), with only minor and evidently transient formation of brushite (calcium phosphate dihydrate; Tansman et al., 2015, 2017b,c). Anecdotal reports from industry and informal observations from our laboratory suggest that these 
surface crystals can grow to sizes that cause a gritty or sandy texture in the rinds of soft, washed-rind cheeses.

Advances in analytical approaches have helped to fuel a growing interest in cheese crystal research, including several recent studies that employed new applications of powder X-ray diffractometry (PXRD; Tansman et al., 2014, 2015, 2017a,b). A limitation of this technique, however, has been the presence of high background noise due to the difficulty of removing noncrystalline cheese material from the harvested crystals, which can make interpretation of X-ray diffraction patterns difficult and result in unidentified crystal phases (Bloss, 2012). This difficulty seems especially problematical when analyzing surface scrapings from soft, washed-rind and bloomy rind cheeses (Tansman et al., 2017a,b).

Polarized light microscopy (PLM), a technique in optical mineralogy and crystallography, is another promising approach that has been recently applied to study cheese crystals (Tansman et al., 2017b,c). Use of PLM offers a simple and inexpensive analytical approach when crystals occur as single crystals because the anisotropic nature of single crystals lends them unique identifying features, such as angle of extinction and birefringence, when viewed using this methodology (Nesse, 2004). Angle of extinction refers to the specific position that some crystals go extinct, or dark/transparent, when viewed under cross-polarized light (Nesse, 2004). Birefringence refers to the coloring some crystals take on when viewed under cross-polarized light. The type and intensity of birefringence can help indicate crystal type (Dyar et al., 2008). Single crystals also tend to exhibit characteristic shapes and geometries that can aid in identification (Bloss, 2012). Image analysis software can be used to quantify the shape and size of crystals, allowing for robust analysis and differentiation. These crystal morphologies, along with intensity of birefringence and angle of extinction, can all be used to tentatively identify specific crystal species.

Previous studies confirmed that the principal surface crystals on soft, washed-rind cheeses, namely ikaite and struvite, occurred as single crystals that were readily observed using PLM under crossed polarizers (Tansman et al., 2017b,c). The present work applies the above criteria to identify single crystals present on the surface of soft, washed-rind cheeses. The specific objectives of this work were (1) to identify surface crystals on soft, washed-rind cheese using specific PLM criteria, (2) to compare PLM identification against PXRD, and (3) to develop and evaluate a novel treatment for cheese surface smear material to improve the analyses of cheese crystals by both PLM and PXRD.

\section{MATERIALS AND METHODS}

\section{Cheeses Samples}

Four different soft, washed-rind cheese varieties were purchased at local retailers in the Burlington, Vermont, area. Two wheels of each cheese variety with identical code date and vat identifier (if available) were obtained and served as duplicate experimental units. All analyses were performed identically on each of the 2 wheels for each of the 4 varieties. All cheese samples were stored at $3^{\circ} \mathrm{C}$ for up to $3 \mathrm{~d}$ until analyses were conducted. A flat-tip pH electrode (Thomas Scientific, Swedesboro, NJ) was used for surface $\mathrm{pH}$ measurements. The probe was placed on 3 separate rind locations (top, side, and bottom) and measurements were averaged. (6 total $\mathrm{pH}$ measurements per cheese variety) Moisture analyses were conducted in triplicate using a forced-draft oven set at $100^{\circ} \mathrm{C}$. A wedge of cheese from each wheel was homogenized using a mortar and pestle. Approximately $2 \mathrm{~g}$ of homogenized sample was weighed into predried and preweighed aluminum pans and then allowed to dry for $24 \mathrm{~h}$ at $100^{\circ} \mathrm{C}$, until a constant weight was reached (6 total moisture content measurements per cheese variety).

\section{Smear Collection}

Smear scrapings were collected from a $1-\mathrm{cm}^{2}$ section of cheese rind using a metal spatula. Care was taken to limit the fracturing of crystals. Scrapings were applied directly to microscope and diffraction slides for PLM and PXRD analyses, respectively, using a metal spatula and a dissection needle.

\section{Alkaline Dispersion}

Alkaline dispersions ( $\mathrm{pH}=10)$ of smears were prepared by submerging a sample of smear (collected as described above) in $15 \mathrm{~mL}$ of $0.0001 \mathrm{M} \mathrm{NaOH}$ (Fisher Scientific, Pittsburgh, PA) in a $50-\mathrm{mL}$ beaker. Beakers were vortexed gently to encourage dispersion of smear matrix. Alkaline mixtures were allowed to sit at room temperature for approximately $3 \mathrm{~h}$. Crystals that settled to the bottom of the beaker were used for PLM and PXRD analyses.

\section{PXRD}

A zero-background diffraction slide was used for all PXRD analyses. Cheese smear scrapings were mounted directly on the slide and overlaid with a thin film of 
mineral oil, as described in previous work (Tansman et al., 2017b,c). For alkaline dispersion samples, 300 $\mu \mathrm{L}$ of crystal-containing smear dispersion liquid (pipetted from the bottom of the beaker) was applied to the diffraction slide. A tissue (Kimwipes, Kimberly-Clark Professional, Roswell, GA) was used to absorb excess liquid. Diffractograms were generated on a Miniflex II powder X-ray diffractometer (Rigaku, The Woodlands, TX) using a speed of $2^{\circ} 2 \theta / \mathrm{min}(2 \theta$ represents the angle formed between the X-ray source, diffraction slide, and X-ray detector) between $5^{\circ} 2 \theta$ and $50^{\circ} 2 \theta$. Diffraction patterns were compared with existing reference diffraction patterns archived in the International Center for Diffraction Data (ICDD) database (http://www .icdd.com). Diffractograms were analyzed and compared using the PDXL diffractometer software package (Rigaku). The following ICDD reference cards were used: \#01-074-7147 (ikaite), \#00-015-0762 (struvite), and \#01-074-3640 (brushite). The experimental diffraction patterns were compared with the reference pattern from each ICDD card to account for all peaks in the experimental diffraction pattern. The absence of additional unexplained peaks indicated all crystal types were elucidated by the reference patterns within the sensitivity of the equipment and experimental design. Some experimental diffraction patterns exhibited preferred orientation, but did not prevent identification of the major crystal phases present in the experimental samples. Preferred orientation results in diffraction patterns with missing peaks or peaks of diminished intensity (Klug and Alexander, 1954).

\section{Polarized Light Microscopy}

Samples collected by the scraping method were applied to a glass microscope slide and overlaid with a thin film of mineral oil to prevent dehydration. Samples prepared in alkaline dispersion were analyzed directly in a beaker to prevent atmospheric exposure of the crystalline entities. Polarized light micrographs were captured using a Nikon E200POL microscope (Nikon Corporation, Tokyo, Japan). Samples were viewed under crossed polarizers. Images were recorded using a SPOT Idea 1.3 MP color camera (SPOT Imaging Solutions, Sterling Heights, MI). The microscope was equipped with a rotating stage, which was used to analyze angles of extinction. Observed objects were determined to be single crystals if they displayed uniform extinction. Amorphous materials and polycrystals will not display uniform extinction and are easily differentiated from single crystals on this basis. Video was captured of each field of view while rotating the stage a full $360^{\circ}$. Angle of extinction measurements were determined using these videos and an on-screen protractor (Protractor for Mac, Trilithon Software, New York, NY). Three fields of view (FOV) were used for each duplicate cheese (6 total images were collected per cheese variety); FOV were chosen at random by moving the stage random distances in the $\mathrm{x}$ and $\mathrm{y}$ directions. Random distances were determined using the "random" package (Eddelbuettel, 2017) in $\mathrm{R}$ (version $\mathrm{R}$ version 3.3.2; R Foundation for Statistical Computing, Vienna, Austria).

\section{Image Analysis}

The PLM images were imported into Adobe Photoshop (Adobe Systems Inc., San Jose, CA), where the crystalline entities were outlined and a black background was applied. Overlapping crystals, crystals with edges obscured by smear material, and crystals intersecting the FOV border were not outlined and not used for any further analysis. The outlined images were analyzed using Fiji (ImageJ distribution, Schindelin et al., 2012). Images were converted to 8-bit black and white, and thresholding was applied using the Li's Minimum Cross Entropy thresholding option ( $\mathrm{Li}$ and Lee, 1993). The threshold images were then analyzed using the particle analysis plugin. Metrics of interest (area and circularity) were collected and recorded for each isolated crystal in each processed image. Outlined entities smaller than 50 pixels $^{2}$ were not included due to limited measurement accuracy when analyzing very small objects.

\section{Statistical Analysis}

Crystal grouping data and crystal harvest technique data were analyzed via a one-way ANOVA. Analyses were performed using JMP Pro 13 (SAS Institute Inc., Cary, NC). A $P<0.05$ level of significance was used for all analyses.

\section{RESULTS AND DISCUSSION}

\section{Cheeses Samples}

All cheese samples had surface $\mathrm{pH}$ values and moisture contents that were consistent with the soft, washed-rind cheese category (Table 1). Surface $\mathrm{pH}$ values varied by approximately 0.5 , and moisture content varied by approximately $5 \%$ across the different cheeses. These observed differences are perhaps a result of differences in make procedure, ripening regimen, and age of the samples at the time of observation. 
Table 1. Cheese sample compositional information; results are from triplicate measurements from duplicate wheels of cheese

\begin{tabular}{lllllll}
\hline & & \multicolumn{2}{c}{ Surface $\mathrm{pH}$} & & \multicolumn{2}{c}{ Moisture content (\%) } \\
\cline { 3 - 4 } \cline { 5 - 6 } Sample & $\begin{array}{l}\text { Manufacture } \\
\text { location }\end{array}$ & Mean & SD & & Mean & SD \\
\hline Cheese A & Vermont & 6.50 & 0.04 & & 47.3 & 0.92 \\
Cheese B & New York & 6.71 & 0.03 & & 45.2 & 0.57 \\
Cheese C & Vermont & 6.84 & 0.04 & & 47.9 & 0.92 \\
Cheese D & Vermont & 7.06 & 0.04 & & 51.9 & 0.85 \\
\hline
\end{tabular}

\section{PLM}

Extinction angle, birefringence, and image analysis results stratified the observed crystals into 2 well-defined groupings (Table 2). Examples of polarized light micrographs of crystals isolated from smear scrapings are shown in Figure 1. Group 1 crystals exhibited an average size and circularity of approximately 5,600 $\mu \mathrm{m}^{2}$ and 0.662 , respectively, and were observed in all 4 cheeses used in this study. These crystals exhibited angles of extinction in the range of $89.5^{\circ}$ to $91.0^{\circ}$ (mean $=90.1^{\circ}$ ) and high birefringent coloring. Group 1 crystals can be seen in Figure 1 marked with hollow arrows. Their birefringence is manifested as bright colors, such as blue, orange, teal, yellow, and green. A common occurrence observed during PLM analyses was a color transformation of group 1 crystals when the microscope stage was rotated. They often started as bright blue and, upon rotation past extinction (i.e., transparency), transitioned to bright orange. The use of the quartz (quarter- $\lambda$ ) filter enhances weak birefringent coloring, and a similar process has been used previously to identify urate crystals, which showed a characteristic color transformation from yellow to blue (Hardy and Nation, 1984). Group 1 crystals had an angle of extinction of approximately $90^{\circ}$, which is known as parallel extinction in the optical mineralogy field (Dyar et al., 2008). The shape of group 1 crystals were often trapezoidal and angular, as confirmed by circularity measurements. Group 1 crystals were significantly smaller and less circular than group 2 crystals $(P<0.05)$. The angle of extinction, shape, and birefringent coloring of group 1 crystals are consistent with the mineral struvite, which exhibits parallel extinction (Ellis, 1962), has a characteristic angular or needle-like shape (Wilsenach et al., 2007), and has similar coloring (Daudon et al., 2016).

The average size and circularity of group 2 crystals was approximately $22,000 \mu \mathrm{m}^{2}$ and 0.708 , respectively, and were observed in cheeses A, C, and D. They were significantly larger and rounder or more circular than group 1 crystals $(P<0.05)$. They had angles of extinction in the range of $13.5^{\circ}$ to $24.5^{\circ}$ as well as little-to-no birefringent coloring (shown in Figure 1, solid arrows). The absence of birefringence displays as crystals having a white coloring. Group 2 crystals exhibited a mean extinction angle of $18.1^{\circ}$. Angle of extinction not equal to $90^{\circ}$ is known as inclined extinction. The range of extinction angles observed for group 2 crystals is expected and is due to random orientation of the crystals along the long growth axis (Bloss, 1961), as well as difficulties during angle of extinction measurement. Round crystals, fractured crystals, and crystals obscured by smear material make proper orientation and angle measurement more difficult. The angle of extinction, shape, and low birefringent coloring of group 2 crystals are consistent with the mineral ikaite, which exhibits inclined extinction at an angle around $17^{\circ}$ (Hesse et al., 1983), has a characteristic round or oval shape, and has similar coloring (Rysgaard et al., 2012).

\section{PXRD}

Diffractograms of smear scrapings (Figure 2) identified crystals in cheeses A, B, and D. Smear scrapings

Table 2. Crystal grouping results from image analysis of polarized light micrographs

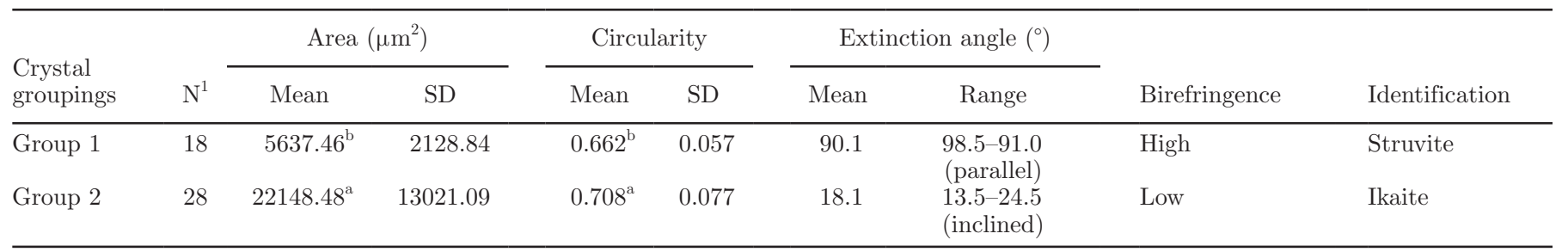

\footnotetext{
${ }^{\mathrm{a}, \mathrm{b}}$ Differing letters within a column indicate a significant difference $(P<0.05)$.

${ }^{1}$ Number of crystals analyzed in each grouping.
} 


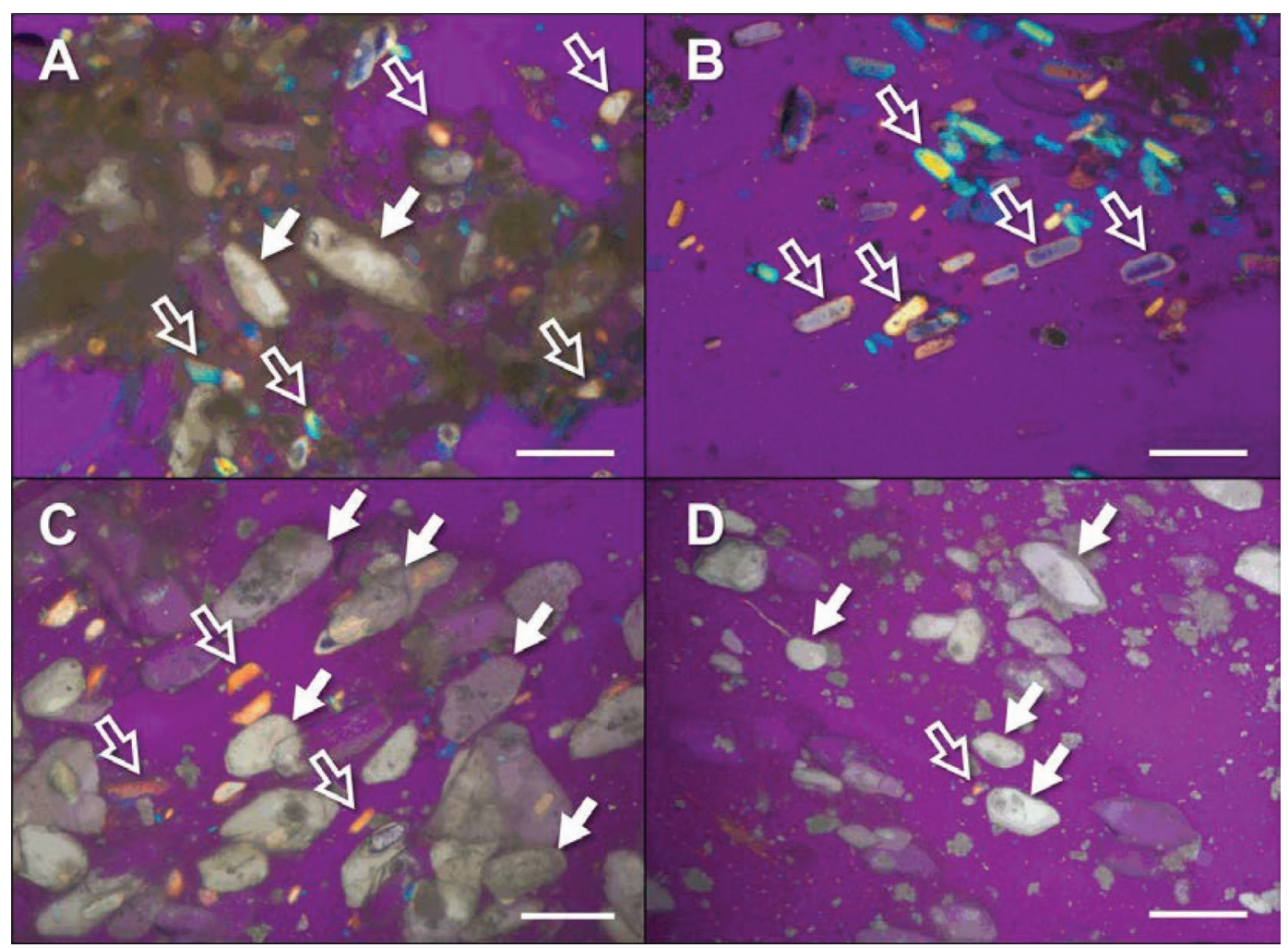

Figure 1. Polarized light micrographs captured via the smear scraping method from cheeses A, B, C, and D. Examples of ikaite crystals labeled with solid arrows. Examples of struvite crystals labeled with open arrows. Scale bars represent $250 \mu \mathrm{m}$. Viewed under $1 / 4 \lambda$ plate (quartz filter). (1/4 $\lambda$ refers to a quarter-wave plate that transforms polarized light into elliptical/circular polarized light, which accentuates birefringent coloring.) Color version available online.

from cheese A displayed distinctive peaks consistent with struvite $\left(\mathrm{NH}_{4} \mathrm{MgPO}_{4} \cdot 6 \mathrm{H}_{2} \mathrm{O}\right)$ and ikaite $\left(\mathrm{CaCO}_{3} \cdot 6 \mathrm{H}_{2} \mathrm{O}\right)$. Cheese $\mathrm{B}$ smear scrapings were found to only contain struvite crystals. Cheese C smear scrapings resulted in diffractograms displaying high amorphous background noise with no identifiable crystal peaks. Cheese D smear scrapings displayed peaks consistent with struvite and ikaite crystals. The absence of discernable crystal peaks in the diffractograms from cheese $\mathrm{C}$ smears (Figure 2C) is at odds with the group 1 and 2 crystals identified as struvite and ikaite, respectively, during PLM analysis (Figure 1C; Table 3). However, all of the diffractograms obtained from the 2 wheels of cheese $\mathrm{C}$ displayed extremely high background noise, even when the analyses were repeated several times. Although high background noise was present, to varying degrees, across all analyzed smear samples, the exceptionally high background noise of cheese $\mathrm{C}$ made complete identification more difficult and increased the possibility of not detecting certain crystalline phases that may have been present in small amounts. This may possibly explain the discrepancy with cheese C, which clearly displayed crystals that were identified as ikaite and struvite by PLM, but which showed no evidence of crystals by PXRD.

\section{Significance of Ikaite, Struvite, and Brushite}

Ikaite, struvite, and brushite have all been previously identified in soft, washed-rind cheese (Tansman et al., $2015,2017 \mathrm{~b}, \mathrm{c})$. These minerals most likely form as a result of physiochemical changes during cheese ripening, including radial mineral migration and gaseous changes of the ripening atmosphere (McSweeney, 2004; Tansman et al., 2017b). The surface pH of washedrind cheeses could encourage the growth and stability of certain crystalline species. Struvite is preferentially crystallized at a pH range of 7.0 to 11.0 (Kim et al., 2016). Crystal formation plays an important role in cheese softening and texture development by sequestering important structural components of the casein matrix, such as calcium and phosphate. In addition to changes of the cheese body, crystal formation may also have an effect on the sensory properties of the cheese surface as well. Anecdotal reports from cheesemakers and mongers point toward surface grittiness potentially 
Table 3. Summary of crystal identification results from powder X-ray diffractometry (PXRD) and tentative identification from polarized light microscopy (PLM)

\begin{tabular}{llll}
\hline & \multicolumn{2}{c}{ PXRD } & \\
\cline { 2 - 3 } Sample & $\begin{array}{l}\text { Smear } \\
\text { scraping }\end{array}$ & $\begin{array}{l}\text { Alkaline } \\
\text { dispersion }\end{array}$ & PLM \\
\hline Cheese A & Struvite & Struvite & Struvite \\
& Ikaite & Ikaite & Ikaite \\
Cheese B & Struvite & Brushite & \\
Cheese C & None identified & Struvite & Struvite \\
Cheese D & Struvite & Struvite \\
& Struvite & Ikaite & Ikaite \\
& Ikaite & Struvite & Struvite \\
& & Ikaite & Ikaite \\
\hline
\end{tabular}

being a common defect among smear ripened cheeses (M. Kehler, Cellars at Jasper Hill, Greensboro, VT, personal communication). Present results suggest that certain crystals, especially ikaite, may grow large enough to surpass the sensory perception threshold and result in grittiness. Vaisey-Genser et al. (1989) estimated the crystal size threshold for grittiness in margarine spreads to be $22 \mu \mathrm{m}$, whereas Hough et al. (1990) noted a detection threshold range of 45 to $105 \mu \mathrm{m}$ for sandiness in Dulce de Leche. A similar threshold of $45 \mu \mathrm{m}$ has been used for the detection threshold of ice crystals in ice cream (Goff and Hartel, 2013). Figure 1 shows many crystals that exceed the length of the scale bars shown $(250 \mu \mathrm{m})$. Crystals of this order of size are very likely exceeding the sensory perception threshold, but further study linking crystal size to sensory response is needed to ascertain the threshold level of grittiness or sandiness in these cheeses.

\section{Crystal Identification via PLM}

Based on the results from PXRD analyses, and the crystal groupings identified from PLM images, we make the supposition that group 1 and 2 crystals can tentatively be identified as struvite and ikaite, respectively. The overall crystal types identified by the different analysis techniques used in the present study are summarized in Table 3. Struvite crystals were significantly smaller and less circular than ikaite crystals (Table 2). Struvite also exhibited high birefringent coloring and parallel extinction, whereas ikaite had low birefringence and inclined extinction (Table 2; Figure 1). These properties are consistent with observations in our previous work (Tansman et al., 2017b,c) and the optical mineralogy literature (Ellis, 1962; Hesse et al., 1983). Crystal size and circularity may change based on the cheese age and crystals fracturing during examination, but angle of extinction and birefringence are intrinsic properties


Figure 2. Powder X-ray diffractometry patterns of crystals isolated from cheeses A, B, C, and D via the smear scraping method. Diffractogram peaks are labeled as being characteristic of ikaite (square) or struvite (triangle). Y-axis uses arbitrary units (AU) of counts per second. ( $2 \theta$ represents the angle formed between the X-ray source, diffraction slide, and X-ray detector). Color version available online.

not dependent on crystal size or shape (Dyar et al., 2008). Although angle of extinction and birefringence are likely sufficient to aid in the identification among the small subset of crystals known to occur on the rinds of smear-ripened cheeses, more advanced optical techniques can be employed. One such technique is to examine interference figures, which appear as regions of light and dark with specific coloring depending on the type of crystal (Bloss, 1961). A Bertrand lens is inserted between the ocular and microscope objective to see an 
interference figure. Interference figures were generated for ikaite and struvite crystals, which were biaxial (-) $2 \mathrm{~V} \sim 40^{\circ}$ and biaxial $(+) 2 \mathrm{~V} \sim 45^{\circ}$, respectively, in agreement with reference values (Hudson Institute of Mineralogy, 2018). This confirms the conclusion made using observations of birefringence, extinction angle, and crystal morphology. These criteria can be used as part of a robust methodology for identifying crystal types via PLM and are standard techniques in the optical crystallographic of minerals in subdisciplines of geology. The small subset of crystals identified via PXRD made positive identification of struvite and ikaite via PLM possible. The use of PXRD should be paired with PLM analysis to ensure all crystal phases are fully accounted for.

Although PXRD will remain the gold standard for crystal identification, it has possible drawbacks. The high capital cost and advanced experience needed to interpret results is often exclusionary for most cheese technology professionals. The presence of amorphous material can also make PXRD analysis difficult and prevent the detection of crystalline entities, as evident in Figure 2C. Furthermore, it is conceivable that amorphous forms of mineral precipitation, such as amorphous calcium phosphate and calcium carbonate, might also occur in cheese smears, which cannot be identified by PXRD (or PLM). Although outside the scope of this project, other analytical techniques could be employed to better detect mineral components. In future work the use of infrared microscopy could allow for the detection of amorphous mineral precipitates that had yet to crystallize. This would lend greater insight into the true scope of mineral migration occurring within these cheese types and the sequence and kinetics of crystallization at the cheese surface.

\section{Alkaline Dispersion Sample Preparation}

As a possible remedy to the high amorphous background issue, alkaline dispersions of each smear scraping were prepared and analyzed via PXRD and PLM. Diffractograms from each alkaline smear dispersion can be seen in Figure 3. Cheese A smear dispersion displayed characteristics of struvite, ikaite, and brushite crystals. Cheese B displayed peaks consistent with only struvite crystals being present. Cheese $\mathrm{C}$ displayed peaks indicative of struvite and ikaite crystals. Cheese D was found to contain struvite and ikaite. Across all diffractograms, less amorphous background noise was present, as can be viewed by the lower baselines in Figure 3. Of note is the emergence of diffractogram peaks corresponding to ikaite and struvite in the alkaline smear dispersion of cheese C (Figure 3C), which

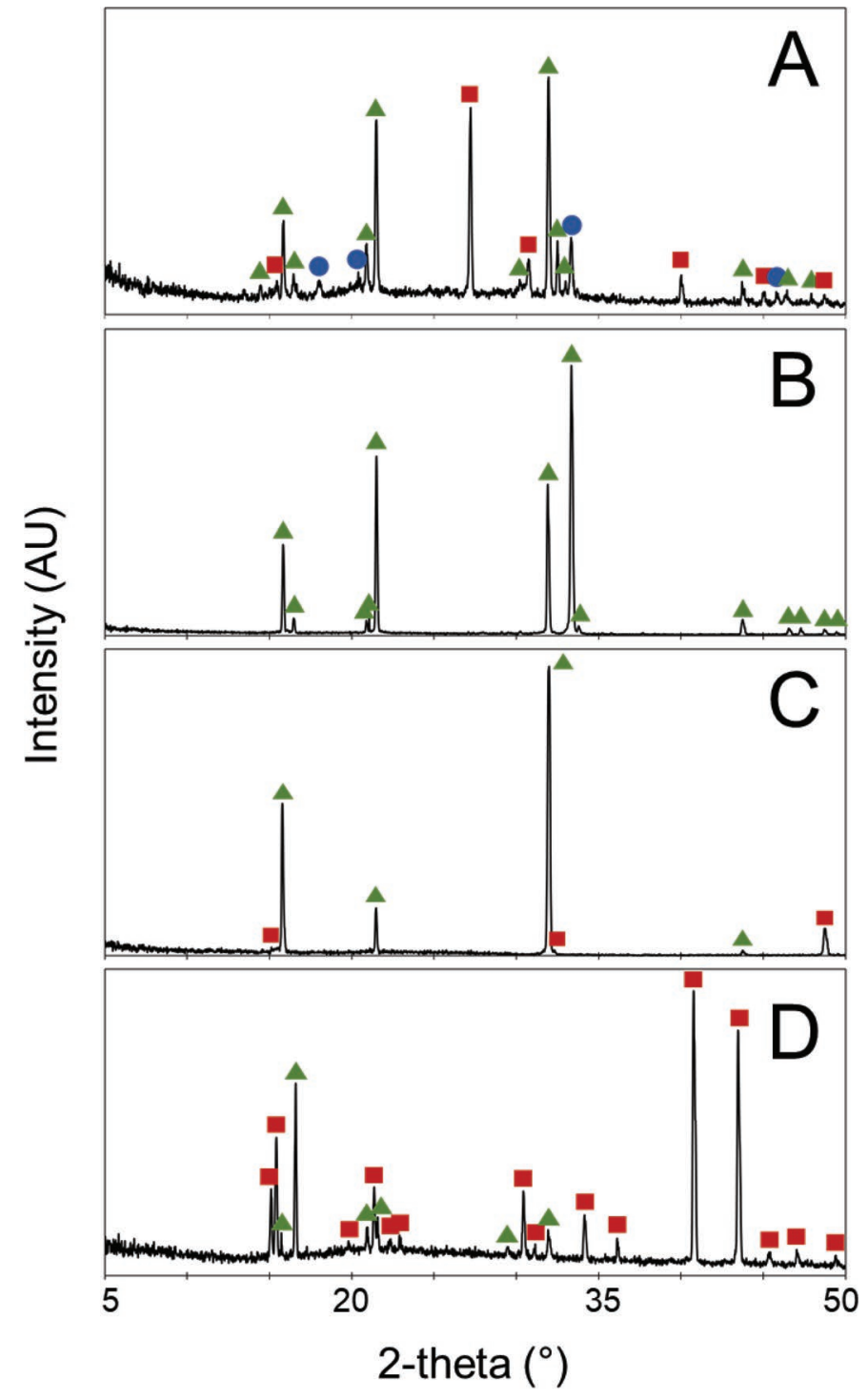

Figure 3. Powder X-ray diffractometry patterns of crystals isolated from cheeses A, B, C, and D via the alkaline dispersion method. Diffractogram peaks are labeled as being characteristic of ikaite (square), struvite (triangle), or brushite (circle). Y-axis uses arbitrary units $(\mathrm{AU})$ of counts per second. ( $2 \theta$ represents the angle formed between the X-ray source, diffraction slide, and X-ray detector). Color version available online.

were consistent with the identifications by PLM, but were not present in the direct smear scrapings (Figure $2 \mathrm{C}$; Table 3 ). This suggests that the alkaline treatment improved the sensitivity of the PXRD identification by separating the smear crystals from the amorphous smear material that caused high baseline noise in the direct smear sample. We also noted the emergence of diffractogram peaks corresponding to brushite in the alkaline dispersion of cheese A. This could be due to the improved PXRD sensitivity, or it could indicate 


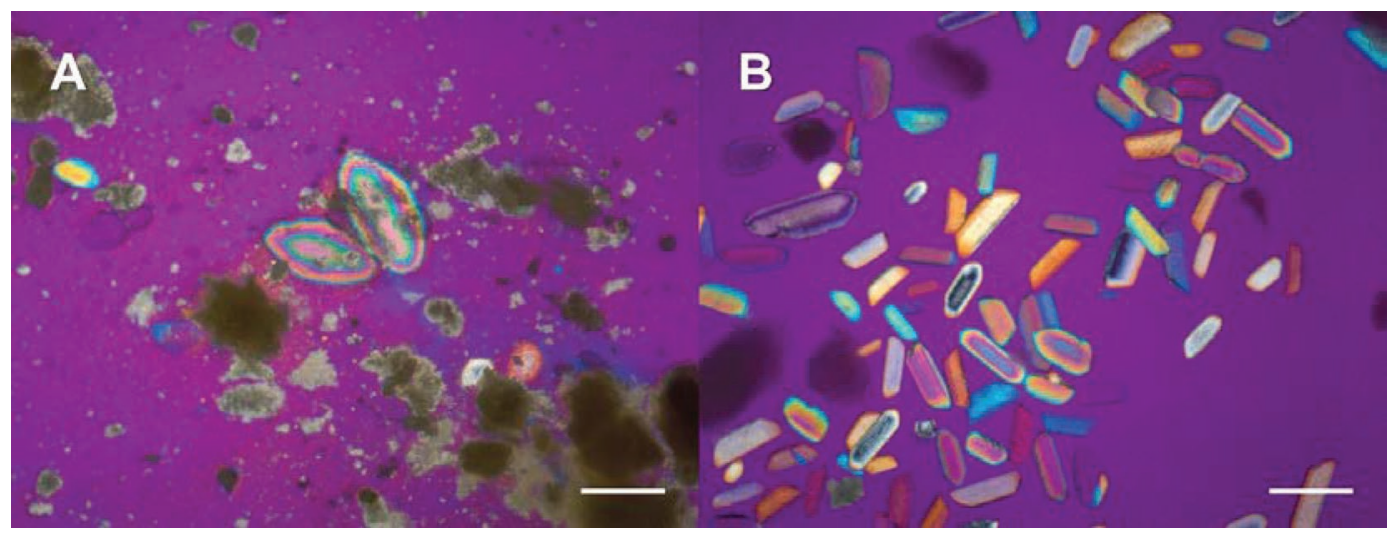

Figure 4. Polarized light micrographs comparing image quality when the smear scraping technique was employed (A) and when the alkaline dispersion method was used (B). Scale bars represent $250 \mu \mathrm{m}$. Viewed under $1 / 4 \lambda$ plate (quartz filter). (1/4 $\lambda$ refers to a quarter-wave plate that transforms polarized light into elliptical/circular polarized light, which accentuates birefringent coloring.) Color version available online.

induced crystallization due to the alkaline environments to which the smears were subjected. Although the latter is possible, the absence of brushite from the 3 other alkaline treatments points toward better PXRD performance as the causative factor. Previous work (Tansman et al., 2017b) has also shown brushite to be endogenous to cheese smears when measured directly, without alkaline treatment.

Summary data of image analysis results from crystals isolated via alkaline smear dispersions, and also via smear scrapings, are shown in Table 4. Crystals analyzed from the alkaline dispersions were smaller and less circular on average than crystals analyzed via the smear scraping method. The alkaline dispersions may have caused the crystals to partially dissolve or may have preferentially separated smaller, less circular crystals. However, the large differences in standard deviations between the 2 treatments violated the assumption of homogeneity of variance, and therefore a statistical difference test could not be performed on mean crystal area and circularity. We found a noticeable improvement of quality of the micrographs captured when the alkaline dispersion method was used (Figure 4). Smear material was present in minute quantities and crystal entities were clearer and more easily identifiable. Thus, the alkaline dispersion technique coupled with PLM

Table 4. Comparison of crystal size and shape between the 2 harvesting techniques

\begin{tabular}{|c|c|c|c|c|}
\hline \multirow{2}{*}{$\begin{array}{l}\text { Extraction } \\
\text { Method }\end{array}$} & \multicolumn{2}{|c|}{ Area $\left(\mu \mathrm{m}^{2}\right)$} & \multicolumn{2}{|c|}{ Circularity } \\
\hline & Mean & $\mathrm{SD}$ & Mean & $\mathrm{SD}$ \\
\hline Alkaline dispersion & $17,738.57$ & $14,690.18$ & 0.632 & 0.110 \\
\hline Smear scraping & 68.923 .06 & $125,800.24$ & 0.700 & 0.026 \\
\hline
\end{tabular}

could be a powerful identification tool, but perhaps not suitable for quantification of crystal size or shape. A possible improvement to this method could be to perform it at lower temperature. If the alkaline dispersion took place at $4^{\circ} \mathrm{C}$, where ikaite is more stable, it may remedy the crystal dissolution and allow for better analysis of ikaite in a native form.

These micrographs also allowed for observations of multigenerational crystal growth and possible resorption (Figure 5). Multigenerational crystal growth can occur after an initial crystal is formed (first generation), and reaction conditions change such that new crystal growth occurs at a future time, using the first generation as a seed crystal. This new growth is known as a second-generation crystal (Klapper, 2010) and may be the result of an influx of minerals necessary for crystal growth to occur again. Resorption occurs when crystals dissolve back into solution, which can be indicative of a shift in equilibrium (Ginibre et al., 2007). A struvite crystal can be seen in Figure 5 with an irregular morphology and pitted surface, suggesting it may be being resorbed into solution. This suggests that changes in the mineral content of the cheese's water phase resulted in struvite being dissolved back into solution, while a second generation of ikaite was crystallizing. Both of these phenomena suggest that the smears on washedrind cheeses are complicated chemical systems that can influence complex crystallization dynamics.

\section{CONCLUSIONS}

The present study outlines a novel approach to analyzing the size and shape of surface-situated crystals on externally bacterial ripened cheeses via PLM and image analysis, as well as a set of criteria to establish the 


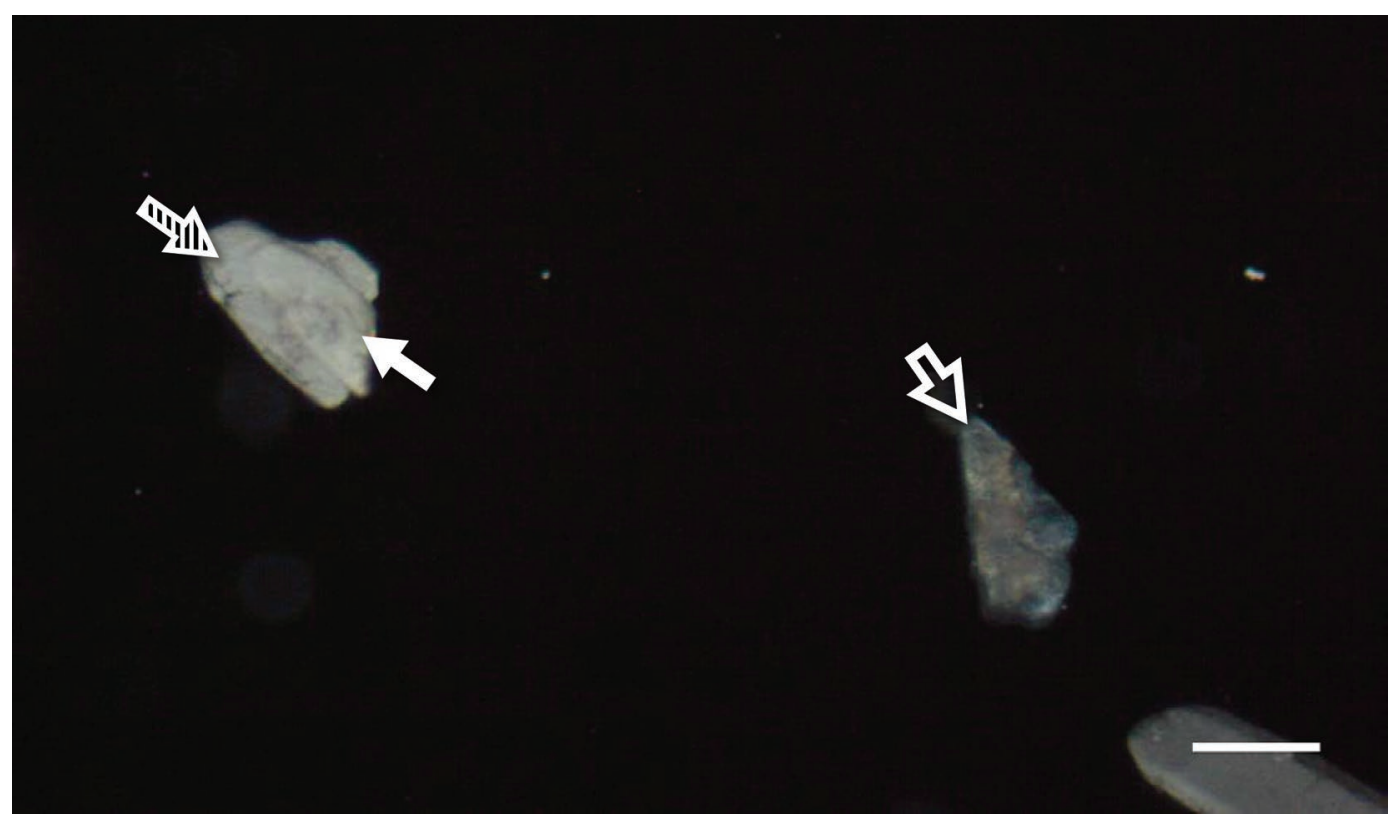

Figure 5. Polarized light micrograph captured using the alkaline dispersion method. Example of ikaite showing multigenerational growth. First generation (solid arrow) can be seen encompassed by second generation (striped arrow). Struvite showing possibility of resorption/dissolving into aqueous phase (open arrow). Scale bar represents $75 \mu \mathrm{m}$. Color version available online.

identity of these crystals. When PXRD analyses are not feasible due to cost or lack of trained personnel, PLM is a powerful tool that can lend valuable insight into the types of crystals present on the surface of smear ripened cheeses. These findings provide exciting opportunities for researchers to further study mineral crystallization in cheese and its effect on sensory properties.

\section{ACKNOWLEDGMENTS}

This study was funded by Hatch Project 033286 (USDA, Washington, DC). The National Science Foundation (Alexandria, VA) is acknowledged for support of grant EAR-0922961 for the purchase of the powder X-ray diffractometer.

\section{REFERENCES}

Bloss, F. D. 1961. An Introduction to the Methods of Optical Crystallography. Holt, Rinehard, and Winston Inc., New York, NY.

Bloss, F. D. 2012. Crystallography and Crystal Chemistry. Hold, Rinehart, Winston, Inc., New York, NY.

Daudon, M., V. Frochot, D. Bazin, and P. Jungers. 2016. Crystalluria analysis improves significantly etiologic diagnosis and therapeutic monitoring of nephrolithiasis. C. R. Chim. 19:1514-1526.

Dyar, M. D., M. E. Gunter, and D. Tasa. 2008. Mineralogy and Optical Mineralogy. Mineralogical Society of America, Chantilly, VA.

Eddelbuettel, D. 2017. random: True Random Numbers using RANDOM.ORG. R package version 0.2.6. Accessed Mar. 5, 2017. https://CRAN.R-project.org/package=random.

Ellis, S. E. 1962. Phosphate minerals in a calculus from the reticuletureen of the sheep. Mineral. Mag. 33:600-608.
Ginibre, C., G. Worner, and A. Kronz. 2007. Crystal zoning as an archive for magma evolution. Elements 3:261-266.

Goff, H. D., and R. W. Hartel. 2013. Ice Cream. 7th ed. Springer, New York, NY

Hardy, R. H., and B. Nation. 1984. Acute gout and the accident and emergency department. Arch. Emerg. Med. 1:89-95.

Hesse, K. F., H. Küppers, and E. Suess. 1983. Refinement of the structure of Ikaite, $\mathrm{CaCO}_{3} \cdot 6 \mathrm{H}_{2} \mathrm{O}$. Z Zeitschrift fur Kristallographie 163:227-231.

Hough, G., E. Martinez, and A. Contarini. 1990. Sensory and objective measurement of sandiness in Dulce de Leche, a typical Argentine dairy product. J. Dairy Sci. 73:604-611.

Hudson Institute of Mineralogy. 2018. Mineral information and data Accessed Feb. 28, 2018. https://www.mindat.org/.

Karahadian, C., and R. C. Lindsay. 1987. Integrated roles of lactate, ammonia, and calcium in texture development of mold surfaceripened cheese. J. Dairy Sci. 70:909-918.

Kim, D., K. J. Min, K. Lee, M. S. Yu, and K. Y. Park. 2016. Effects of $\mathrm{pH}$, molar ratios and pre-treatment on phosphorus recovery through struvite crystallization from effluent of anaerobically digested swine wastewater. Environ. Eng. Sci. 21:12-18.

Klapper, H. 2010. Generation and propagation of defects during crystal growth. Pages 92-132 in Springer Handbook of Crystal Growth. Springer, Berlin, Germany.

Klug, H. P., and L. E. Alexander. 1954. X-ray Diffraction Procedures: For Polycrystalline and Amorphous Materials. Vol. 2. Wiley, New York, NY.

Le Graet, Y., and G. Brule. 1988. Migration des macro et oligo-éléments dans un fromage à pâte molle de type Camembert. Lait $68: 219-234$

Le Graet, Y., A. Lepienne, G. Brule, and P. Ducruet. 1983. Migration du calcium et des phosphates inorganiques dans les fromages à pâte molle de type Camembert au cours de l'affinage. Lait 63:317-332.

Li, C. H., and C. K. Lee. 1993. Minimum cross entropy thresholding. Pattern Recognit. 26:617-625.

McSweeney, P. 2004. Biochemistry of cheese ripening. Int. J. Dairy Technol. 57:127-144. 
Metche, M., and J. Fanni. 1978. Rôle de la flore fongique dans l'accumulation du calcium et du phosphore à la surface des fromages du type camembert. Lait 58:336-354.

Nesse, W. D. 2004. Introduction to Optical Minerology. 3rd ed. Oxford University Press, New York, NY.

Rysgaard, S., R. N. Glud, K. Lennert, M. Cooper, N. Halden, R. J. G. Leakey, F. C. Hawthorne, and D. Barber. 2012. Ikaite crystals in melting sea ice-Implications for $\mathrm{pCO} 2$ and $\mathrm{pH}$ levels in Arctic surface waters. Cryosphere 6:901-908.

Schindelin, J., I. Arganda-Carreras, E. Frise, V. Kaynig, M. Longair, T. Pietzsch, S. Preibisch, C Rueden, S. Saalfeld, B. Schmid, J. Y. Tinevez, D. J. White, V. Hartenstein, K. Eliceiri, P. Tomancak, and A. Cardona. 2012. Fiji: an open-source platform for biologicalimage analysis. Nat. Methods 9:676-682

Tansman, G. F., P. S. Kindstedt, and J. M. Hughes. 2014. Powder $\mathrm{X}$-ray diffraction can differentiate between enantiomeric variants of calcium lactate pentahydrate crystal in cheese. J. Dairy Sci. 97:7354-7362.

Tansman, G., P. S. Kindstedt, and J. M. Hughes. 2015. Crystal fingerprinting: Elucidating the crystals of Cheddar, Parmigiano-Reg- giano, Gouda, and soft washed-rind cheeses using powder x-ray diffractometry. Dairy Sci. Technol. 95:651-664.

Tansman, G., P. S. Kindstedt, and J. M. Hughes. 2017a. Crystallization and demineralization phenomena in stabilized white mold cheese. J. Dairy Sci. 100:6074-6083.

Tansman, G., P. S. Kindstedt, and J. M. Hughes. 2017b. Crystallization and demineralization phenomena in washed-rind cheese. J. Dairy Sci. 100:8694-8704.

Tansman, G., P. S. Kindstedt, and J. M. Hughes. 2017c. Minerals in food: Crystal structures of ikaite and struvite from bacterial smears on washed-rind cheese. Can. Mineral. 55:89-100.

Vaisey-Genser, M., B. K. Vane, and S. Johnson. 1989. Graininess, crystal size, and firmness of stored canola oil margarines. J. Texture Stud. 20:347-361.

Wilsenach, J. A., C. A. H. Schuurbiers, and M. C. M. van Loosdrecht. 2007. Phosphate and potassium recovery from source separated urine through struvite precipitation. Water Res. 41:458-466. 